\title{
The rare patient with infrainguinal stent restenosis a complica- tion: Atherectomy device fracture
}

\author{
Metin Onur Beyaz ${ }^{\text {a, }}$, Burcu Sadikoglu Lale ${ }^{\text {a }}$, Sefer Kaya ${ }^{a}$, Dilan Oruç ${ }^{a}$, Serhat Hakkoymaz ${ }^{\text {, }}$, Se- \\ nem Urfali ${ }^{\mathrm{b}}$, Iyad Fansa ${ }^{\mathrm{a}}$ \\ ${ }^{a}$ Department of Cardiovascular Surgery, Tayfur Ata Sokmen Faculty of Medicine, Hatay Mustafa Kemal University, Hatay, Turkey. \\ ${ }^{b}$ Department of Anesthesiology and Reanimation, Tayfur Ata Sokmen Faculty of Medicine, Hatay Mustafa Kemal University, Hatay, \\ Turkey.
}

\begin{abstract} during peripheral artery atherectomy.

Keywords: Peripheral artery disease; atherectomy; foreign body
\end{abstract}

Excisional atherectomy alone or followed by balloon angioplasty in patients with infra-inguinal peripheral artery disease is an acceptable and appropriate treatment strategy. In this article, a case study of a 67-year-old man who had an intravascular foreign body removed due to an aterectomy device fracture while performing atherectomy due to peripheral arterial disease in the Cardiovascular Surgery Unit of our hospital is presented. The foreign body was demonstrated by a digital subtraction angiography examination. Access to the superficial femoral artery was made by an incision on the anterior $1 / 3$ of the right thigh, and the broken atherectomy part was excised. Surgical intervention may be required to remove the intravascular foreign body that is broken

\section{INTRODUCTION}

Peripheral artery disease (PAH) is a clinical condition that has been increasing in recent years. Although it has various clinical variations for itself, the majority of patients suffer from claudication which affects their lifestyle ${ }^{[1]}$. In addition to the medical and surgical approach, angiographic treatment methods, especially infrainguinal atherectomy, and additional balloon angioplasty have been widely used ${ }^{[2]}$. Cilostazol which has clearly proven efficacy in medical treatment can't be used in patients with left ventricular failure. That is why we use endovascular methods like angioplasty, atherectomy, or a combination of these in patients with infra-inguinal peripheral artery disease ${ }^{[3]}$. Performing angioplasty to the superficial femoral artery (SFA) is

\footnotetext{
* Corresponding author: Metin Onur BEYAZ

Mailing address: Departmant of Cardiovascular Surgery, Tayfur Ata Sokmen Faculty of Medicine, Hatay Mustafa Kemal University, Hatay, Turkey.

E-mail: metinonurbeyaz@gmail.com

Received: 22 March 2021 / Accepted: 24 January 2022
}

generally an inadequate treatment. It is characterized by a high degree of calcification of the SFA and the widespread involvement of the disease and a much higher prevalence of occlusion than stenosis ${ }^{[4]}$. There are a number of atherectomy devices for SFA disease including rotational atherectomy, orbital atherectomy, and excisional. There is no comparison has been made between available atherectomy devices in randomized studies ${ }^{[5]}$. There is no clear signal of superiority among these treatment modalities in observational studies. Thus, the choice of device is left to the experience and expertise of the surgeon.

We present a case of a 67-year-old male, who was previously treated with an infrainguinal stent in an external center. The stent was totally cluded and we were planned to treat using excisional atherectomy and balloon angioplasty.

In this case, the blood vessel was occluded within 3 months following stenting. It is unique in that the atherectomy catheter is broken in the patient scheduled for atherectomy treatment.

In this case report, the mechanism and management of the complication are discussed. 


\section{CASE REPORT}

A 67-year-old male patient was admitted to our clinic with the complaint of right calf claudication, which has been progressive for the last 4 weeks and restricted his lifestyle. There was no history of ischemic rest pain or non-healing wounds. On the right, he had an ABI (Anke Brachial Index) of 0.6.

His past medical history was remarkable in terms of coronary artery disease, tobacco use, and femoropopliteal bypass above the knee with PTFE graft to the same extremity and peripheral artery stenting below the inguinal ligament. Diagnostic angiography showed extensive right iliac artery disease, total occlusion in the common femoral artery and right SFA, and old nitinol stents totally occluded in both the common femoral artery (CFA) and distal of the SFA. After consultation with the patient, it was decided to continue endovascular treatment of the right external iliac, CFA, and SFA. The left common femoral artery access is done without any complications. A long angiographic sheet was placed into the left iliac artery and it was advanced in a retrograde way until it reached the external right iliac artery. Lesions in the external iliac and femoral arteries were crossed with a $0.18 \mathrm{~mm}$ nitinol wire. We advanced it with the wire of the atherectomy device. Occlusions within the common femoral artery and distally to the external iliac artery were successfully passed with atherectomy (Figure 1). When the distal of the SFA was reached, the atherectomy catheter was broken in the old nitinol stent, which was understood to be subintimal after the procedure.

The patient was operated under general anesthesia under emergency conditions. Under fluoroscopy, the tip of the atherectomy catheter was found and marked. The skin was passed subcutaneously, SFA was found and returned. Transverse arteriotomy was performed after $1 \mathrm{cc}$ heparinization. The broken end of the atherectomy device $(18 \mathrm{~cm})$ was grasped with forceps and taken out. Proximal and distal embolectomy was performed. Inflow and run-off current was seen. The artery was primarily repaired with 0.6 prolene. (Figure 2).

\section{DISCUSSION}

There are other potential complications of atherectomy, but the fracture is rare. There are studies in the literature in which the guide wires were broken independently of atherectomy ${ }^{[6]}$. The most unique feature of our case is that it did not contain the total occlusion of the old nitinol stents before atherectomy, and unfortunately, the old stent placed by another hospital was positioned out of the lumen.

Contact between the incisor of the atherectomy device and the subintimal stent might be among the factors that contributed to this complication, because of loss of structural strength. Various methods have been reported for retrieving broken devices using techniques such

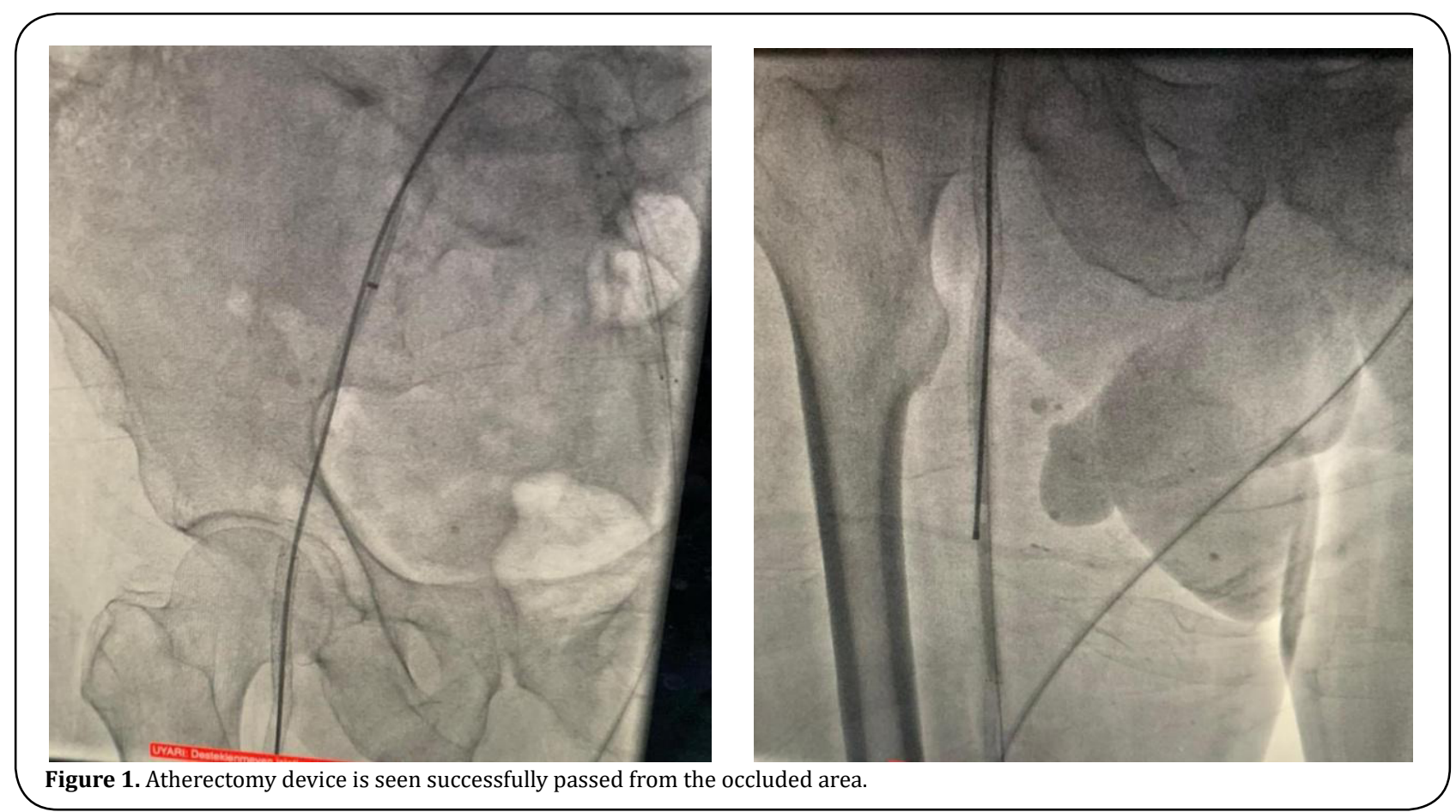



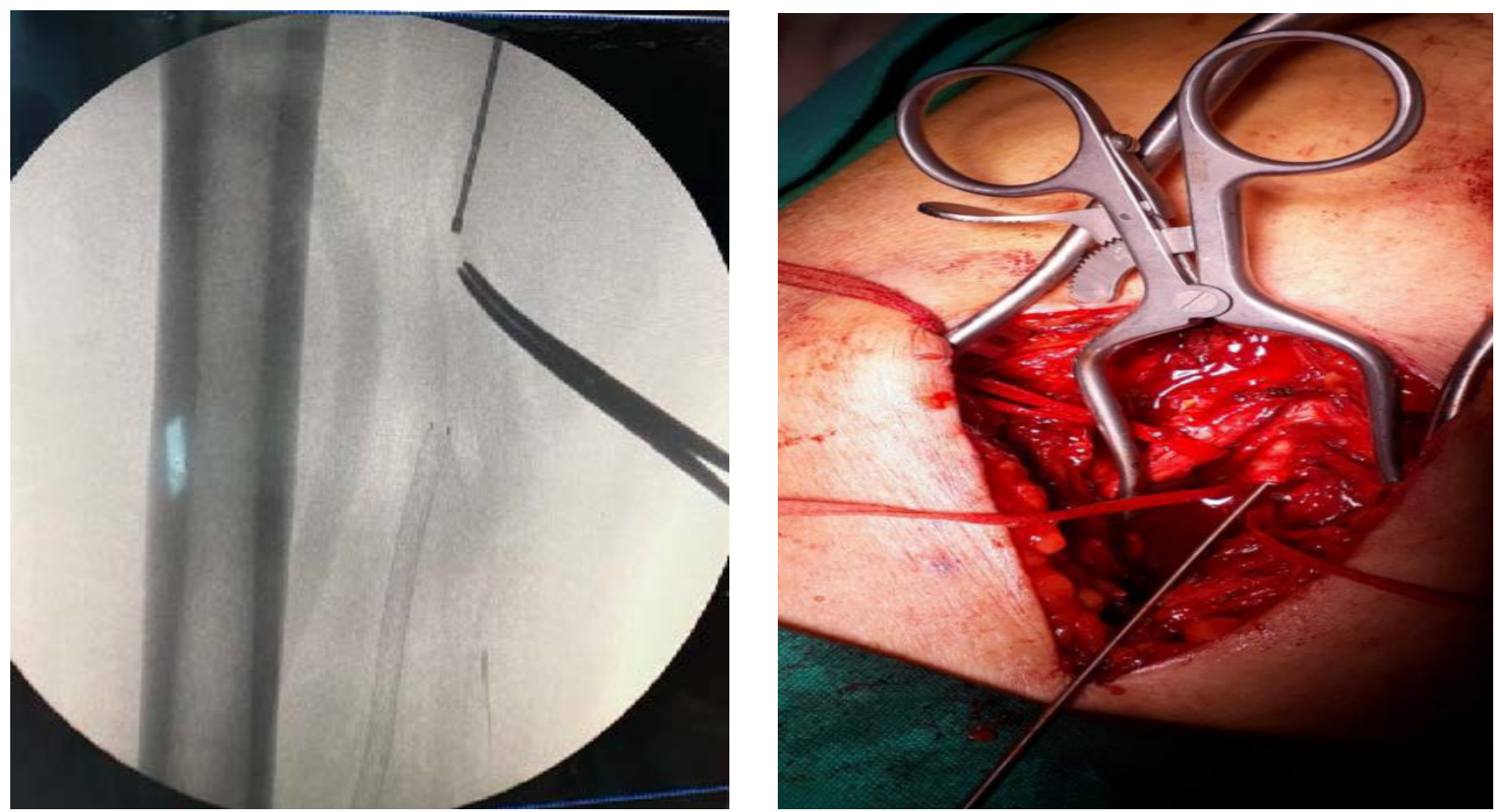

Figure 2. Broken device seen on flouroscopy and incision made on projection of SFA.

as catheter use, biopsy forceps, and balloon inflation ${ }^{[7]}$. In our case, the use of a trap atherectomy catheter was not appropriate because the severed piece was quite long $(18 \mathrm{~cm})$. In addition, it was thought that the pressure balloon inflation technique was not applicable due to the risk of rupture of the vessel wall by the very hard foreign body. Although the guidewire technique is theoretically possible to retrieve the split end, the capture guidewires will not always be entangled. With endovascular methods and the use of infra-inguinal stents, there is a success rate above 95\%. However, because of the nitinol stent structure, the risk of subintimal placement, and the movements of the limbs, the chance of success decreases to $50 \%{ }^{[8]}$. Because of these risks, atherectomy and balloon angioplasty are thought to be more appropriate in stents in the infra inguinal artery ${ }^{[9]}$.

\section{CONCLUSION}

The use of atherectomy should be reconsidered in cases with infrainguinal stent implantation due to the subintimal location of the SFA stent and the risk of more risky complications of the atherectomy device.

\section{DECLARATIONS}

\section{Authors' contributions}

Made substantial contributions to conception and de- sign of the study and performed data analysis and interpretation: Beyaz MO, Sadikoglu Lale B, Kaya S;

Performed data acquisition, as well as provided administrative, technical, and material support: Oruc D, Hakkoymaz S, Urfalı S.

Availability of data and materials

All data of the patients are stored at Hatay Mustafa Kemal University where the operations are performed.

\section{Conflicts of interest}

The authors declared that there are no conflicts of interest to disclose.

\section{Ethical approval and consent to participate}

We studied in accordance with the ethical guidelines set by the Helsinki Declaration and the International Association of Heart and Lung Transplantation (ISHLT). A retrospective study was made by obtaining signed documents and approvals from all patients for procedures, including the approval of the use of patient data in future retrospective studies.

\section{REFERENCES}

1. Campia, U., Gerhard-Herman, M., Piazza, G., \& Goldhaber, S. Z. (2019). Peripheral Artery Disease: Past, Present, and Future. American Journal of Medicine, 132(10), 1133-1141.

2. Firnhaber, J. M., \& Powell, C. S. (2019). Lower Extremity Peripheral Artery Disease: Diagnosis and 
Treatment. American Family Physician, 99(6), 362369.

3. Tsigkou, V., Siasos, G., Rovos, K., Tripyla, N., \& Tousoulis, D. (2018). Peripheral artery disease and antiplatelet treatment. Current Opinion in Pharmacology, 39, 43-52.

4. Tang, Q. H., Chen, J., Hu, C. F., \& Zhang, X. L. (2020). Comparison Between Endovascular and Open Surgery for the Treatment of Peripheral Artery Diseases: A Meta-Analysis. Annals of Vascular Surgery, 62, 484-495.

5. Safian, R. D., Niazi, K., Runyon, J. P., Dulas, D., Weinstock, B., Ramaiah, V., \& Heuser, R. (2009). Orbital atherectomy for infrapopliteal disease: device concept and outcome data for the OASIS trial. Catheterization and Cardiovascular Interventions, 73(3), 406-412.

6. Tsujimura, T., Ishihara, T., Iida, O., Asai, M., Masuda, M., Okamoto, S., ... \& Mano, T. (2019). Successful percutaneous retrieval of a detached microcatheter tip using the guide-extension catheter trapping technique: A case report. Journal of Cardiology Cases, 20(5), 168-171.

7. Gallagher, K. A., Meltzer, A. J., Ravin, R. A., Graham, A., Shrikhande, G., Connolly, P. H., ... \& McKinsey, J. F. (2011). Endovascular management as first therapy for chronic total occlusion of the lower extremity arteries: comparison of balloon angioplasty, stenting, and directional atherectomy. Journal of Endovascular Therapy, 18(5), 624-637.

8. Schillinger, M., Sabeti, S., Dick, P., Amighi, J., Mlekusch, W., Schlager, O., ... \& Minar, E. (2007). Sustained benefit at 2 years of primary femoropopliteal stenting compared with balloon angioplasty with optional stenting. Circulation, 115(21), 27452749.

9. Clegg, S., Aghel, A., \& Rogers, R. K. (2014). Late presenting, contained rupture of the superficial femoral artery following atherectomy and stenting: case report and literature review. Catheterization and Cardiovascular Interventions, 84(2), 257-261. 